\title{
Filling in the Roadmap for Self-Consistent Electron Cloud and Gas Modeling*
}

\author{
J.-L. Vay ${ }^{\dagger}$, M. A. Furman, P. A. Seidl, LBNL, CA, USA \\ R. H. Cohen, A. Friedman, D. P. Grote, M. Kireeff Covo, A. W. Molvik, LLNL, CA, USA \\ P. H. Stoltz, S. Veitzer, Tech-X Corporation, USA \\ J. Verboncoeur, UC Berkeley, USA
}

\begin{abstract}
Electron clouds and gas pressure rise limit the performance of many major accelerators. A multi-laboratory effort to understand the underlying physics via the combined application of experiment, theory, and simulation is underway. We present here the status of the simulation capability development, based on a merge of the three-dimensional parallel Particle-In-Cell (PIC) accelerator code WARP and the electron cloud code POSINST, with additional functionalities. The development of the new capability follows a "roadmap" describing the different functional modules, and their inter-relationships, that are ultimately needed to reach self-consistency. Newly developed functionalities include a novel particle mover bridging the time scales between electron and ion motion, a module to generate neutrals desorbed by beam ion impacts at the wall, and a module to track impact ionization of the gas by beam ions or electrons. Example applications of the new capability to the modeling of electron effects in the High Current Experiment $(\mathrm{HCX})$ are given.
\end{abstract}

\section{INTRODUCTION}

Electron clouds and gas pressure rise limit the performance of many major accelerators [1]. A multi-laboratory effort to understand the underlying physics via the combined application of experiment, theory, and simulation is underway. The development of the simulation capability follows a "roadmap" describing the different functional modules, and their inter-relationships, that are ultimately needed to reach self-consistency.

The new capability development is based on a merge of the three-dimensional parallel Particle-In-Cell accelerator code WARP [2] and the electron cloud code POSINST $[3,4]$, with additional functionalities. POSINST has been developed for E-cloud studies in high-energy accelerators or storage rings such as the APS $\left(\mathrm{e}^{+} / \mathrm{e}^{-}\right.$, short bunches $\sim 1$ $\mathrm{cm}$, well-separated $\sim 0.85-100 \mathrm{~m}, C \sim 1.1 \mathrm{~km}$, intense $N \sim 5 \times 10^{10}$, high-energy $\left.E \sim 7 \mathrm{GeV}, \gamma \sim 14,000\right)$ or the PSR (single long proton bunch, $\sim 60 \mathrm{~m}, C=90$ m, intense $N \sim 5 \times 10^{13}$, low-energy $E \sim 1.7 \mathrm{GeV}$, $\gamma=1.85$ ). WARP is a multidimensional intense beam simulation program being developed and used by the Heavy Ion Fusion (HIF) Virtual National Laboratory [5], whose

${ }^{*}$ This work was performed under the auspices of the U.S. Department of Energy by University of California, LLNL and LBNL under contracts W-7405-Eng-48, and DE-AC03-76F00098.

$\dagger$ jlvay@lbl.gov goal is to develop heavy-ion accelerators capable of igniting inertial-fusion targets for electric-power production, as well as heating matter for the study of high-energy density physics. A set of parameters for accelerators currently in service or being considered as steps on the development path toward HIF are: HCX, or High Current Experiment, (one $\mathrm{K}^{+}$beam, $180 \mathrm{~mA}, 1.0 \mathrm{MeV}, 4 \mu$ s pulse length); driver $\left(120 \mathrm{Bi}^{+}\right.$beams, $1 \mathrm{~A}-2 \mathrm{kA}, 1.6 \mathrm{MeV}-4 \mathrm{GeV}, 30$ ms-10 ns pulse length). The HCX, currently in operation at Lawrence Berkeley National Laboratory, is the first transport experiment using a driver-scale heavy-ion beam [6]. It is designed to address important science questions involving the optimum beam size and the preservation of good beam quality during transport, while driver is the fullpower accelerator needed to ignite the DT capsules.

The two codes possess complementary capabilities that are necessary but not sufficient for self-consistent simulations of HIF beams and their interactions with electron cloud and desorbed gas. Newly developed functionalities include a novel particle mover bridging the time scales between electron and ion motion, a module to generate neutrals desorbed by beam ion impacts at the wall, and a module to track impact ionization of the gas by beam ions or electrons.

This paper presents the "roadmap" describing the different functional modules, and their inter-relationships, that are ultimately needed to reach self-consistency, the two codes WARP and POSINST that form the core of the new simulation capability, and new additional functionalities. Example applications of the new capability to the modeling of electron effects in HCX are given.

\section{THE “ROADMAP”}

We have established a list of different functional modules, and their inter-relationships, that are ultimately needed to reach self-consistency for the modeling of HIF beams with e-cloud and gas, and have summarized it in a block diagram (see Fig. 1). We can imagine this as a "roadmap" that we need to follow in order to develop our simulation tools. The most basic block of the roadmap is a self-consistent PIC module that follows the beam through an accelerator lattice with its self-field and images at the wall. Ions from halo that strike the wall can desorb neutrals and electrons that have enough time to reach the beam before the end of the pulse, and interact with it. The time-dependent motion of neutrals and electrons must be tracked, then. The gas can be ionized by beam ions and electrons, and can produce new electrons and ions that must 
be tracked as well. All these particles can hit the walls and produce more neutrals and electrons. Finally beam ions can reflect at the wall, and charge-exchange can occur in the gas. More details on the anaysis that led to the establishment of this roadmap can be found in $[7,8,9,10]$.

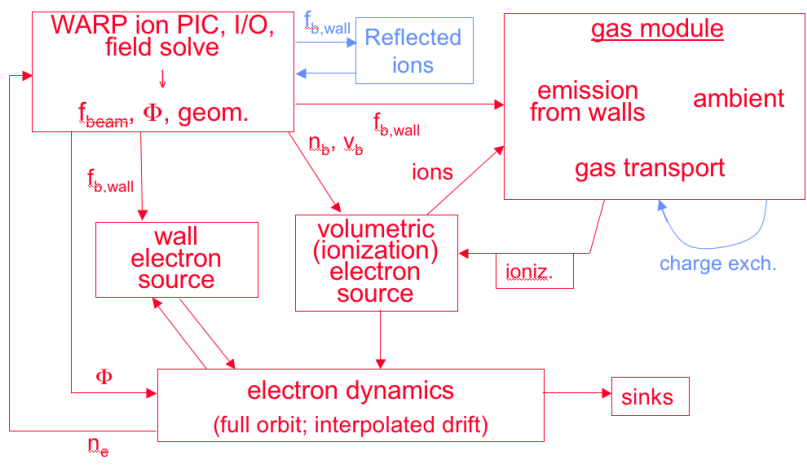

Figure 1: "Roadmap" describing the different functional modules, and their inter-relationships, that are ultimately needed to reach self-consistency for the modeling of HIF beams with e-cloud and gas. At the time of this writing, most modules are operational, excepting the "reflected ions" and the "charge exchange" modules that are still being developed.

\section{DESCRIPTION OF WARP AND POSINST}

\section{WARP}

WARP [2] is being designed and optimized for heavy ion fusion accelerator physics studies. It allows flexible and detailed multi-dimensional modeling of high current beams in a wide range of systems, including bent beam lines using a "warped" coordinate system (from which the code derives its name). At present it incorporates a 3-D description, an axisymmetric $(r, z)$ description, a transverse slice $(x, y)$ description, a simple envelope model used primarily to obtain a well-matched initial state, and envelope/fluid models used for scoping and design.

The discrete-particle models in WARP combine the particle-in-cell (PIC) technique commonly used for plasma modeling with a description of the "lattice" of accelerator elements. In 3-D and R-Z, WARP is a time-dependent plasma code - the particles are advanced in time and the self and applied fields are applied directly to update the particles' momenta. The calculation can follow the timedependent evolution of beams, or can efficiently be used to study steady-state beam behavior in 3-D or 2-D R-Z by solving for the self-consistent field only infrequently or by using an iterative method. The transverse-slice model is $\mathrm{s}$-dependent, and is effectively a steady-flow model ( $\mathrm{s}$ is the axial coordinate). The beam can be initially generated from one of several general distributions or from first principles via space-charge-limited injection from an emitting surface.
The self-consistent field is assumed electrostatic Poisson's equation is solved on a Cartesian mesh that moves with the beam. In a bend, the solution is altered to include the curvature of the coordinates. Complex conductor geometry can be included in the field solution using a subgrid-scale, or "cut-cell," boundary algorithm to afford a realistic description of the geometry while minimizing the required grid resolution. Regions where the physics or the geometry require a small spatial scale can be resolved as finely as needed using Adaptive Mesh Refinement [11]. In addition, a specialized refinement patch accommodates space-charge-limited injection with very fast rise time; near the emitting surface, the self-fields are calculated along independent one-dimensional lines normal to the surface, with increasing refinement towards the surface [11].

A general set of finite-length, possibly overlapping, accelerator elements can be specified, including quadrupoles, dipoles, accelerating gaps, and elements with arbitrary multi-pole content, using a MAD-like syntax. Individual elements can be defined and chained together into aggregate elements, which can be further combined. A tool is provided which converts a MAD format file into a WARPreadable file. The fields of the elements can be specified at any of several levels of detail. At the simplest level, the applied fields are axially uniform within hard-edged regions, and "residence corrections" are used in the particle mover so that the particles receive a correct impulse from each element independent of the number of times they "land" within the element on discrete time steps. At the next level, the fields are expressed as axially dependent multipole components. At the most detailed level, the fields are represented on three-dimensional grids. Electrostatic elements can be included from first principles via inclusion of the conductor geometry as a boundary condition in the solution of the self-fields. Another set of elements in the 3-D and slice models specifies the locations and curvatures of bends. These bends are not physical elements but are the appropriate coordinate transformations needed to follow the beam around the bends. WARP handles particle collisions with any object in the beam line, including the pipe wall, beam source components, diagnostics (Faraday cups, etc.).

\section{POSINST}

POSINST [3] models a thin slice of electrons at a fixed location in the machine subject to their own self-field, and to the field created by the bunches passing through it. The distribution of electrons is modeled as a collection of macro-particles while the effect of the chargedparticle bunches is modeled as a chain of external, predefined, kicks applied to the macro-electrons. The possible sources of macro-electrons are: (1) photoelectron emission, (2) secondary electron emission, (3) residual gas ionization, and (4) stray beam particles striking the vacuum chamber walls. The macro-electron self-field is computed 
by summing the contribution from the charges from either all macro-electrons, or from the charge on the nodes of a grid on which the electronic charge density has been deposited. The boundary condition is either open or a perfectly conducting pipe (surface charges included) with elliptical or rectangular geometry, and a possible antechamber.

The secondary electron emission routines developed in POSINST, and repackaged into the CMEE package [12] that is distributed by Tech-X, are based on a detailed probabilistic model of the secondary emission process and are described in detail in Ref. [4]. The model relies on a broad phenomenological fit to data for the secondary emission yield (SEY) and the emitted-energy spectrum, which are used as inputs to the particle simulation.

\section{NEW FEATURES}

\section{New interpolated mover}

Self-consistent simulation of electrons and ions requires simulation of electrons in the quadrupole magnets as well as in the gaps between magnets, and running the simulation long enough to simulate the passage of the ion beam. This results in a broad range of time scales, ranging from the electron cyclotron period $\left(10^{-11}-10^{-10} \mathrm{~s}\right)$ through the ion beam transit time $\left(10^{-7}-10^{-5} \mathrm{~s}\right)$ through a fringe field or a series of lattice elements. The shortest electron cyclotron period is typically one to two orders of magnitude shorter than the next-shortest timescale, usually the electron bounce time in the combined beam-potential and magnetic wells.

To deal with this large range of time scales in a unified manner, we have developed a mover for electrons that interpolates between full electron dynamics and drift kinetics. Specifically, it interpolates in the velocity perpendicular to the magnetic field. Schematically, the velocity is updated in a conventional manner,

$\mathbf{v}_{\text {new }}=\mathbf{v}_{\text {old }}+\Delta t\left[\left(\frac{d \mathbf{v}}{d t}\right)_{\text {Lorentz }}+(1-\alpha)\left(\frac{d \mathbf{v}}{d t}\right)_{\mu \nabla B}\right]$

and the particle position is updated using an effective velocity which is an interpolation of this updated velocity and the drift velocity:

$$
\mathbf{v}_{\mathrm{eff}}=\mathbf{b}(\mathbf{b} \cdot \mathbf{v})+\alpha \mathbf{v}_{\perp}+(1-\alpha) \mathbf{v}_{d} .
$$

The first equation denotes an update of the velocity under the combined influence of electric and magnetic fields (Lorentz force), to which is added a rotation of the velocity in the plane of $\mathbf{v}$ and $\mathbf{B}$ such as to effect the $\mu \nabla B$ acceleration of the parallel velocity that is needed in drift kinetics ( $\mu$ is the magnetic moment). In the second equation, $\mathbf{v}_{d}$ denotes the drift velocity (sum of electric and magnetic drifts), $\alpha$ is an interpolation parameter, and $b=\mathbf{B} / B$. For the particular choice of interpolation parameter $\alpha=$ $1 /\left[1+\left(\omega_{c} \Delta t / 2\right)^{2}\right]^{1 / 2}$, the radius of the gyration motion is physically correct for large as well as small $\omega_{c} \Delta t$. The drift is physically correct as the drift component of $\mathbf{v}$ is $\mathbf{v}_{d}$. Finally, the parallel dynamics is correct as the full particle push in the direction of the magnetic field is retained along with the $\mu \nabla B$ correction. The algorithm is presented in more details in Ref. [8]. This new interpolation scheme preserves a physical gyroradius for any value of $\omega_{c} \Delta t$, which is a significant improvement over the standard Boris mover [13] which causes particles to gyrate with a radius that is large compared to the physical gyro orbit at large $\omega_{c} \Delta t$, as noted in Ref. [14]. Thus, it is well suited for simulating particles that move through regions of strong, weak, and no magnetic field such as we have in HIF accelerators.

\section{Gas module}

Impact of energetic ions with surfaces can lead to desorption of neutrals. At high energies, characteristic of the HIF application, electronic sputtering is the dominant mechanism. In electronic sputtering, the incident energetic ion transfers kinetic energy to electrons in the medium, which transport the energy to the surface and to the impurities adsorbed in the lattice. The desorption yield depends on energy and angle of impact, as well as material properties and surface history.

Because the dependence of the yield is difficult to characterize for real surfaces, in the initial model the yield at normal incidence is specified by a phenomenological quantity $Y_{0}$. The angular dependence of the yield is then characterized by the impact angle $\theta$, where $\theta$ is measured from the surface normal. The enhancement of the yield at large $\theta$ (near grazing incidence) is possibly due to the enhanced backscatter of ions, as calculated by Molvik using SRIM [9]. These backscattered ions result in an increase over the normal yield $Y_{0}$ by nearly a factor of 2 near grazing incidence,

$$
\frac{Y(\theta)}{Y_{0}}=1+1.82 \times 10^{-4} \exp (5.16 \theta)
$$

where $\theta$ is here measured in radians.

The model for the energy and angular distribution of the desorbed neutrals is based on molecular dynamics calculations. The energy dependence in this model is taken from a classical calculation [15]:

$$
f(E)=C_{1} \frac{2 U E}{E_{\mathrm{exc}}(E+U)^{3}},
$$

where $U$ is the surface binding energy, $E_{\text {exc }}$ is the excitation energy, and $C_{1}$ is a normalization constant. The excitation energy can be estimated from

$$
E_{\mathrm{exc}} \approx \frac{d E / d x}{n \pi r_{\mathrm{cyl}}^{2}}
$$

where $d E / d x$ is the stopping power, $n$ is the atomic density at the surface, and $r_{\text {cyl }}$ is the radius for energy deposition. This model was verified experimentally by Berhold 
and Wucher [16]. The cumulative distribution function is given by

$$
F(E)=\frac{\int_{0}^{E} f\left(E^{\prime}\right) d E^{\prime}}{\int_{0}^{\infty} f\left(E^{\prime}\right) d E^{\prime}} .
$$

Inverting for $E$, we can obtain the equation to generate Eq. 4 from a sequence of uniformly distributed random numbers, $0<R<1$ :

$$
\frac{E}{U}=\frac{R+R^{1 / 2}}{1-R} .
$$

Similarly, the angular dependence is taken to be [17]:

$$
f(\theta)=C_{2} \cos ^{2}(\theta),
$$

where $C_{2}$ is a normalization constant. Because of the difficulty in inverting Eq. 8 analytically, we invert it via a Monte Carlo rejection scheme. First, an angle is obtained from a uniformly distributed random number $R 1_{i} \in(0,1)$, $\theta_{i}=180\left(R 1_{i}-1 / 2\right)$. Next, a second random number $R 2_{i}$ is compared to the distribution at $\theta_{i}$; if $R 2_{i} \leq \cos ^{2}\left(\theta_{i}\right)$ then the angle is accepted, otherwise it is rejected. The neutrals created by the gas modules are injected into the simulation as macroparticles and their trajectories, including their interaction with the walls, are tracked using WARP's particle pusher.

\section{Ionization module}

The gas produced by the gas module can be ionized by the beam ions or by electrons. We track these events using the following procedure:

1. deposit the gas density onto a grid $n_{g}$,

2. lookup from $n_{g}$ the local gas density $n_{p}$ at particle positions,

3. compute the number of events $N=n_{p} \sigma v \Delta t$ occuring during the last time step $\Delta t$, where $\sigma$ is the crosssection of the event and $v$ is the relative velocity between the incident particles and the gas,

4. get $N_{\text {int }}=\operatorname{Int}(N)$ and $N_{\text {frac }}=N-N_{\text {int }}$, respectively the integer and the fractional parts of the number of events,

5. pick a random number $0<R<1$; if $R<N_{\text {frac }}$, increment $N_{\text {int }}$ by 1 ,

6. for each of the $N_{\text {int }}$ events, create macro-ions and macro-electrons resulting from ionization.

We have assumed that the gas reservoir is large enough and the cross-section for gas ionization is small enough that the depletion of gas due to ionization can be neglected.

Since it is computationally costly to generate random numbers, and since the probability of ionizing gas is small for each time step, the array of incident particles is scanned with a stride $s$ that is set by the user, and multiplying $N$ by $s$. Typical values for $s$ are $10<s<100$. In order to ensure that the whole array is scanned after $s$ time steps, the scan starts at position $\bmod (i, s)$ at the $i^{\text {th }}$ iteration.

\section{MODELING OF THE HCX MAGNETIC SECTION}

We study electron effects in the magnetic section of HCX [18], shown in Fig. 2. A suppressor ring electrode, surrounding the beam after it exits the last quadrupole magnet, can be biased to $-10 \mathrm{kV}$ to prevent ion-induced electron emission off an end wall (a slit plate) from reaching the magnets, or can be left unbiased to allow electrons to be emitted from the end wall and to flow into the magnets. There is also a series of three clearing electrodes, labeled (a), (b) and (c) on Fig. 2, in the drift regions between quadrupole magnets, which can be biased positively to draw off electrons from between any pair of magnets.

The current that flows in and out of these clearing electrodes is monitored in the experiment and is compared with simulations in Fig. 3, in the case where the suppressor ring electrode was left grounded to allow electrons to propagate upstream, and the three clearing electrodes were biased to $+9 \mathrm{kV}$. For convenience, we label the electrons created by the beam hitting the end wall as "primary", while we label the electrons created by the primary electrons hitting the vacuum pipe surrounding the magnets as "secondary". Two simulations were performed, one with secondary electrons OFF and one with secondary electrons ON.

The primary electrons created at the end plate and propagating upstream can enter only two quadrants of the fourth (last) magnet, because of the sign of the $\mathbf{E} \times \mathbf{B}$ drift, and then drift upstream. It is expected that most of the electrons that reached the entrance of the fourth magnet will be lost in the clearing electrode (c) which is biased at +9 $\mathrm{kV}$. Applying the same reasoning to electrodes (b) and (a), it is expected that the current collected will be larger on (c) than (b), and (b) than (a). The first assumption is indeed confirmed by both the experiment and the simulations where the averaged current collected on (c) is about ten times that collected on (b). We also note that the role of the secondary electrons is vital in the simulation to recover the experimental signal. On the other hand, while less current is collected on (a) than on (b) in the simulations as originally expected, more current is collected in the experiment. We also observe that while the agreement is better for (b), there is less current collected in the simulation just after the head and at the tail of the pulse. We believe that these discrepancies are due to a combination of beam halo scraping and gas ionization. Measurements are underway to identify causes and adjust the simulation parameters accordingly, making use of the newly developed gas and ionization modules.

\section{CONCLUSION}

We are near completion of a new capability that follows a "roadmap" describing the different functional modules, and their inter-relationships, that are ultimately needed to reach self-consistent modeling of ion beams with e-clouds and gas. Newly developed functionalities include a novel 
particle mover bridging the time scales between electron and ion motion, a module to generate neutrals desorbed by beam ion impacts at the wall, and a module to track impact ionization of the gas by beam ions or electrons. Comparisons of simulations using the new capability with HCX measurements have provided encouraging results and we are working toward getting even better agreement using the newly developed modules. The new capability is also being applied to the modeling of high-energy physics accelerators [19].

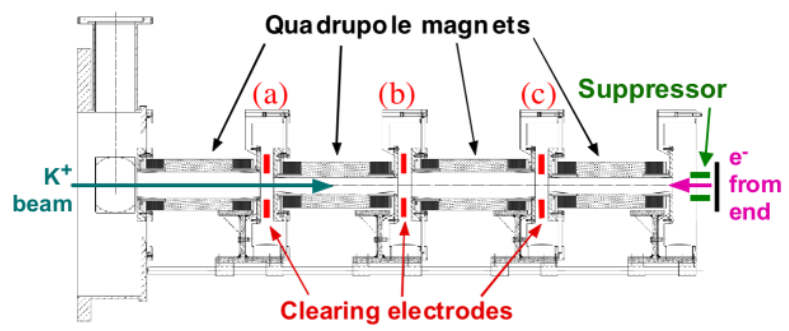

Figure 2: HCX in region of 4 quadrupole magnets, with clearing electrode rings between magnets and a suppressor electrode ring after the last magnet.

\section{REFERENCES}

[1] Proc. 31st ICFA Advanced Beam Dynamics Workshop on Electron-Cloud Effects (ECLOUD04), Napa, CA, USA, 1923 Apr 2004, CERN Report CERN-2005-001 (2005), ISBN 92-9083-241-X, http://icfa-ecloud04.web.cern.ch/icfaecloud04/agenda.html

[2] D. P. Grote, A. Friedman, J.-L. Vay. I. Haber, AIP Conf. Proc. 749, 55 (2005)

[3] M. A. Furman and G. R. Lambertson, Proc. Intl. Workshop on Multibunch Instabilities in Future Electron and Positron Accelerators "MBI-97," KEK, p. 170; M. A. Furman, LBNL-41482/LHC Project Report 180, May 20, 1998.

[4] M. A. Furman and M. T. F. Pivi, PRSTAB/v5/i12/e124404 (2003).

[5] G. Logan, et al, Nuclear Fusion 45, 131 (2005).

[6] L. R. Prost, P. A. Seidl, F. M. Bieniosek, C. M. Celata, A. Faltens, D. Baca, E. Henestroza, J. W. Kwan, M. Leitner, W. L. Waldron, R. Cohen, A. Friedman, D. Grote, S. M. Lund, A. W. Molvik, and E. Morse, PRSTAB 8, 020101 (2005).

[7] R. H. Cohen, A. Friedman, S. M. Lund, A. W. Molvik, E. P. Lee, T. Azevedo, J.-L. Vay, P. Stoltz, S. Veitzer, PRSTAB 7, 124201 (2004).

[8] R. H. Cohen, A. Friedman, M. Kireeff Covo, S. M. Lund, A. W. Molvik, F. M. Bieniosek, P. A. Seidl, J.-L. Vay, P. Stoltz, S. Veitzer, Phys. of Plasmas 12 (2005)

[9] A. W. Molvik, M. Kireeff Covo, F. M. Bieniosek, L. Prost, P. A. Seidl, D. Baca, A. Coorey, and A. Sakumi, PRSTAB 7, 093202 (2004).

[10] P. H. Stoltz, M. A. Furman, J. L. Vay, A. W. Molvik and R. H. Cohen, PRSTAB 6, 054701 (2003).
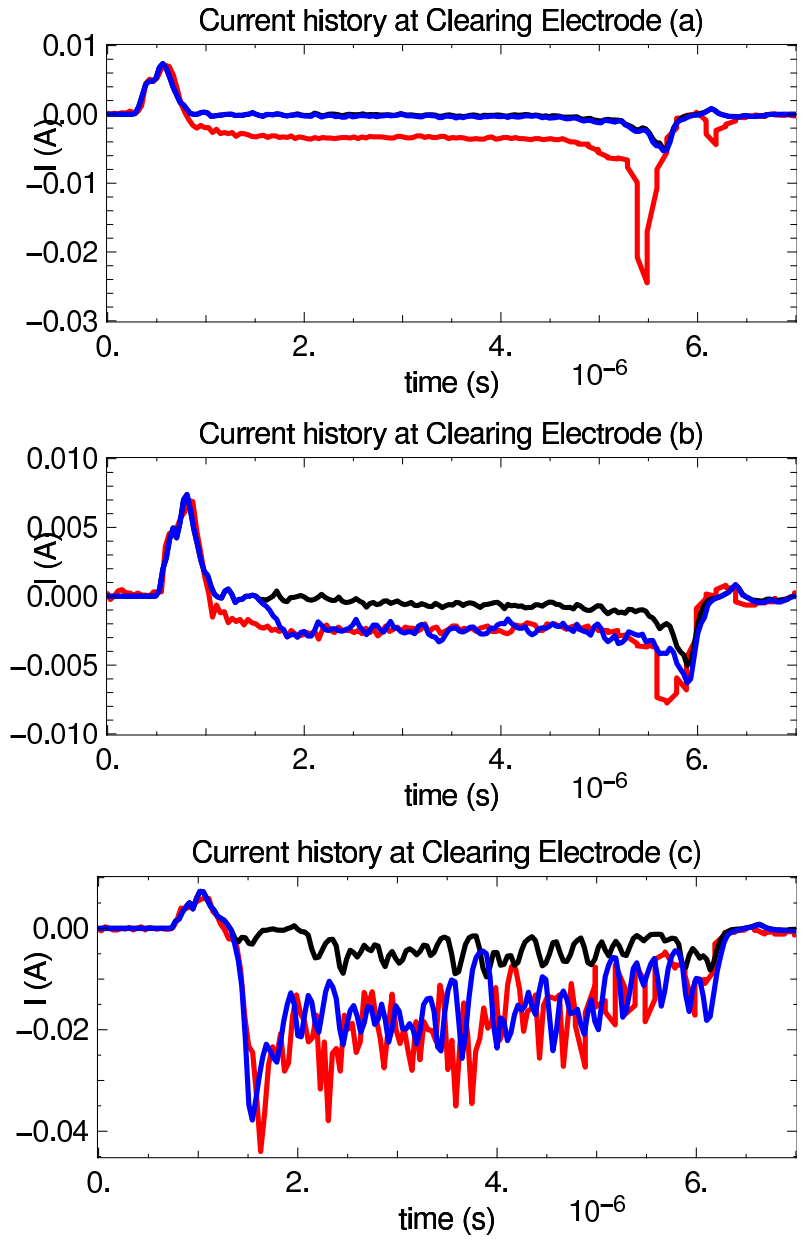

Figure 3: Current history at clearing electrodes (a), (b) and (c): red - recorded on HCX experiment, black - WARP simulation with secondary electrons OFF, blue - WARP simulation with secondary electrons $\mathrm{ON}$.

[11] J.-L. Vay, P. Colella, J. W. Kwan, P. McCorquodale, D. B. Serafini, A. Friedman, D. P. Grote, G. Westenskow, J.C. Adam , A. Heron, I. Haber, Phys. of Plasmas 11, 2928 (2004).

[12] http://www.txcorp.com/technologies/CMEE

[13] J. P. Boris, Proc. 4th Int. Conf. on Num. Sim. of Plasmas, Washington, DC, 2-3 Nov., 1970 (NRL, Washington, 1970; U.S. Gov. Printing Office, Stock Nb 0851 00059).

[14] S. E. Parker and C. K. Birdsall, J. Comp. Phys 97, 91 (1991).

[15] M. W. Thompson, Philosophical Magazine 18, 377 (1968).

[16] W. Berthold and A. Wucher, Physical Rev. B56, 4251 (1997).

[17] E. M. Bringa, R. E. Johnson, and L. Dutkiewicz, Nucl. Instr. and Meth. in Phys. Res. B152, 267-290 (1999).

[18] A.W.Molvik, M. Kireeff Covo, A. Friedman, R. Cohen, S.M. Lund, J.J. Barnard, F. Bieniosek, P. Seidl, D. Baca, J.-L. Vay, C.M. Celata, W.L. Waldron, J.L. Vujic, paper ROPB002, these proceedings.

[19] J.-L. Vay, M. A. Furman, R. H. Cohen, A. Friedman, D. P. Grote, paper FPAP016, these proceedings. 